\title{
Mechanical model of nanoparticles for material removal in chemical mechanical polishing process
}

\author{
Hao CHEN, Dan GUO*, Guoxin XIE, Guoshun PAN \\ State Key Laboratory of Tribology, Tsinghua University, Beijing 100084, China \\ Received: 04 March 2016/Revised: 21 April 2016/Accepted: 05 May 2016 \\ (C) The author(s) 2016. This article is published with open access at Springerlink.com
}

\begin{abstract}
Chemical mechanical polishing (CMP) is the most effective method for surface planarization in the semiconductor industry. Nanoparticles are significant for material removal and ultra-smooth surface formation. This research investigates the mechanical effects of the material removal in the CMP process. The various contact states of pad, individual particle, and wafer caused by the variations of working conditions and material properties are analyzed. Three different mechanical models for the material removal in the CMP process, i.e., abrasive wear, adhesive wear, and erosive wear are investigated, with a focus on the comparison of the results for different models. The conclusions and methods obtained could potentially contribute to the understanding and evaluation of the CMP process in further work.
\end{abstract}

Keywords: nanoparticle; chemical mechanical polishing (CMP); contact theory; material removal

\section{Introduction}

The application of chemical mechanical polishing (CMP) to polish various materials (e.g., metals, glasses and stones) has a long history. In 1965, Monsanto first applied CMP process to the manufacture of glasses. In the 1980s, the International Business Machines Corporation (IBM) produced 4M and 64M Dynamic Random Access Memory (DRAM) [1, 2] by applying the CMP process. This move led to the rapid development of the technique. The CMP process is now widely used in the semiconductor industry for oxide dielectric and metal layer planarization, such as integrated circuit (IC) fabrication for various materials [3]. Accordingly, CMP is the most effective method used in surface planarization.

The recent line width in IC fabrication has the tendency to be $14 \mathrm{~nm}$ and smaller. This measurement leads to a higher requirement for the chip surface planarization process. Therefore, studies on CMP theory and principle have increasingly been performed to develop the control of such a technique.

* Corresponding author: Dan GUO.

E-mail: guodan26@mail.tsinghua.edu.cn
In 1927, Preston [4] proposed a mechanical model to describe the material removal rate (MRR) in CMP. The MRR in his research was a linear correlation with polishing pressure $(P)$ and polishing velocity $(U)$. Warnock [5] put forward a theory involving the geometry effect in CMP. Runnels and Eyman [6] analyzed the fluid film between the wafer and the pad, and demonstrated that hydroplaning was possible for standard CMP processes. Tseng and Wang [7] proposed that MRR $=K P^{5 / 6} U^{1 / 2}$ according to Hertz theory. Zhang and Busnaina [8] pointed out that plastic deformation was the most likely deformation mechanism, which occurred within the abrasive nanoparticle and the wafer during CMP. They also found that $\mathrm{MRR}=K(P U)^{1 / 2}$. Shi and Zhao $[9,10]$ demonstrated that $\mathrm{MRR}=K\left(P^{2 / 3}-P_{\mathrm{th}^{2 / 3}}\right) U$ for critical pressure when the pad was soft. These models are called phenomenological models. They, somehow, match the empirical data but their mechanism is unknown.

Studies on the theoretical contact model have also been conducted. $\mathrm{Yu}$ et al. [11] proposed material removal theory related with pad roughness. Liu et al. [12] analyzed the material removal mechanism when the nanoparticle scrolled between the pad and the 


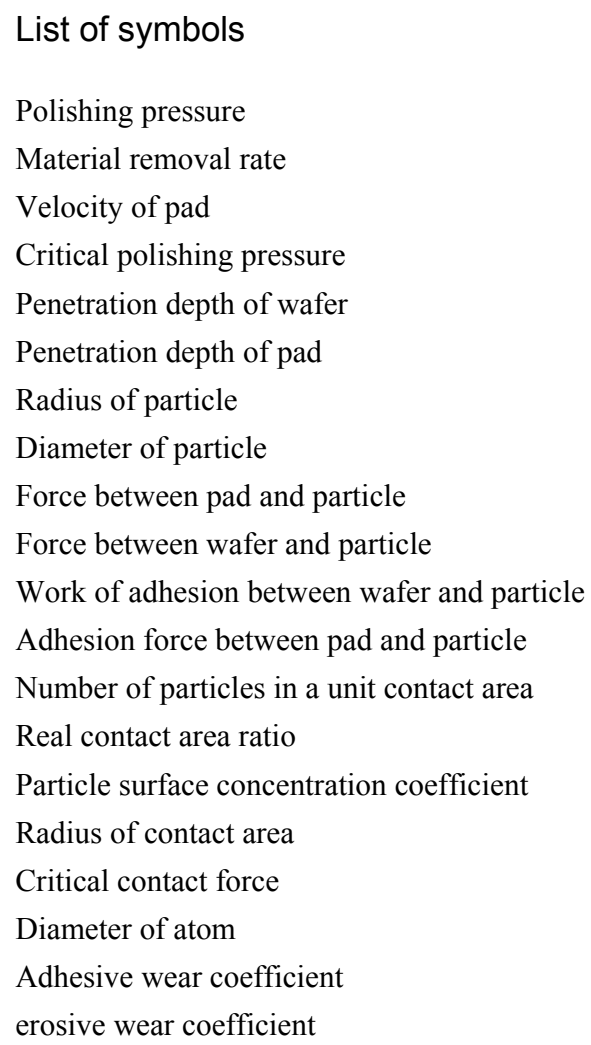

$\begin{array}{clc}P & \text { Composite Young's modulus of pad and particle } & E_{\mathrm{sp}} \\ \text { MRR } & \text { Composite Young's modulus of wafer and particle } & E_{\mathrm{sw}} \\ U & \text { Young's modulus of particle } & E_{\mathrm{s}} \\ P_{\mathrm{th}} & \text { Young's modulus of pad } & E_{\mathrm{p}} \\ \delta_{\mathrm{w}} & \text { Young's modulus of wafer } & E_{\mathrm{w}} \\ \delta_{\mathrm{p}} & \text { Poisson ratio of wafer } & \mu_{\mathrm{w}} \\ r & \text { Poisson ratio of pad } & \mu_{\mathrm{p}} \\ D & \text { Poisson ratio of particles } & \mu_{\mathrm{s}} \\ F_{\mathrm{sp}} & \text { Surface hardness of wafer } & H_{\mathrm{w}} \\ F_{\mathrm{sw}} & \text { Work of adhesion between pad and particle } & W_{\mathrm{ap}} \\ W_{\mathrm{aw}} & \text { Adhesion force between wafer and particle } & F_{\mathrm{aw}} \\ F_{\mathrm{ap}} & \text { Number of adhesion process per second } & n \\ N & \text { mass of a particle } & m \\ k_{\mathrm{r}} & \text { velocity of particle } & v \\ C & \text { Number of impact between per second } & n^{*} \\ a & \text { Roughness of pad } & R_{\mathrm{a}} \\ F_{\mathrm{sp}}^{\mathrm{c}} & \text { Strain energy of wafer } & E_{\mathrm{strain}} \\ d & \text { Ratio of transferred kinetic energy to strain energy } & \eta \\ k_{\mathrm{a}} & \text { Angle of impact direction } & \theta \\ k_{\mathrm{e}} & & \end{array}$

wafer. Zhang and Tanaka [13] focused on the silicon monocrystal deformation induced by two- and three-body contact sliding. Base and Liang [14] thought that mechanical wear by the abrasive nanoparticle was the main cause of the material removal in CMP. Zhao et al. [15] presented an elastic-plastic asperity microcontact model for the contact between two nominally flat surfaces. Luo and Dornfeld [16] found that plastic deformation occurred between the nanoparticles and the wafer. Zhao and Chang [17] supposed that the asperity summits of the pad were random, and the deformation between the nanoparticles and the pad was elastic.

In addition, adhesion or impact between the nanoparticle and the wafer was thought to be a form of material removal in CMP. Ahmadi and Xia [18] studied the material removal by adhesive and abrasive wear during the CMP process. Fu et al. [19] suggested that the hydroxylated layer behavior was modeled as a perfectly plastic material. Wang et al. [20] also proposed a novel mathematical model for CMP based on interface solid physical and chemical theory, which considered abrasive and adhesive wear, in addition to energy equilibrium knowledge. Jeng and Huang $[21,22]$ investigated the effects of particle size within the CMP process using a microcontact wear model, which considered the impact of particle to the wafer surface. $\mathrm{Xu}$ et al. [23, 24] performed an experiment, wherein a cylindrical liquid jet containing $\mathrm{SiO}_{2}$ nanoparticle normally impacted on a glass surface at speeds of $1 \mathrm{~m} / \mathrm{s}$ and $3 \mathrm{~m} / \mathrm{s}$, to evaluate the contribution of the nanoparticle impact to the material removal.

Compared with the phenomenological models [6-12], contact theories and models are more effective in revealing the material removal mechanism in the CMP process. However, only a few studies focused on the removal models of erosive wear, which may be a significant form of material removal [25]. Furthermore, the removal rates of different removal models have not been compared as far. The contact state of the particle, wafer, and pad is also significant for the material removal in CMP. The contact state is also influenced by many factors, including particle diameter, mechanical properties of the pad or wafer, and load on particles. However, the description of the contact state has not been sufficiently considered. 
Two types of particle, wafer, and pad contact states are further discussed in this paper. A theoretical method, which has been used before, is also demonstrated to quantitatively analyze the contact state. Three different removal models, namely, abrasive, adhesive, and erosive wear, are proposed and analyzed. The MRR owing to nanoparticle impact to the wafer is evaluated through equivalent assumption of strain energy to kinetic energy. Comparisons of each removal model are proposed for different pressures, pad velocities, and particle sizes. The conclusion and methods in this paper may help to better understand and evaluate the CMP process in future work.

\section{Theory and analysis}

\subsection{Different contact states}

During the CMP process, not all abrasive particles have the chance to be in contact with the wafer surface. In most situations, only those in the slurry trapped in between the pad asperity summits and the wafer during the CMP process are effective (Fig. 1).

The particle behavior and load acting on each particle vary depending on the slurry concentration and the mechanical properties of the pad and the wafer. All these different factors will lead to two different contact states.

In the CMP process involving a hard pad, the pad deformation is usually small, and the pad function is to hold the abrasive particles [26]. This is the first type of contact state, called the "particle non-embedded". In this situation, the polishing pressure effect mainly transfers through the contact area between the particles and the wafer surface. Therefore, all the pad

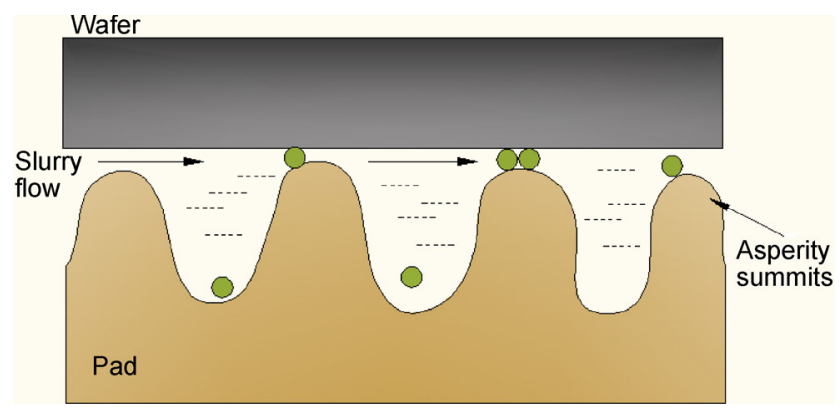

Fig. 1 Schematic of the polishing pad-wafer contact in CMP. pressures change into load on each particle. On the contrary, in the CMP process involving a soft pad, the particles seem to be engulfed because of the larger pad deformation. Hence, most of the pressure acts directly on the contact area between the wafer and the pad. This type is called the "particle embedded," which is in contrast to the first type of contact state. Figure 2 shows the geometrical characteristics of the two contact states.

\subsection{Force analysis for a single particle between the wafer and the pad}

The contact can usually be simplified into a threebody contact of the wafer particle and the polishing pad [13]. The particle in such a model is regarded as a sphere. As shown in Fig. 3, the contact force leads to a penetration depth in the wafer when a particle comes into contact with the water surface. $\delta_{\mathrm{w}}$ is the penetration for the wafer, while $\delta_{\mathrm{p}}$ is that for the pad. $F_{\mathrm{sp}}$ is the force between the pad and the particle, while $F_{\text {ap }}$ is the adhesion force between the two. $F_{\mathrm{sw}}$ is the force between the wafer and the particle, while $F_{a w}$ is the adhesion force between the two.

The force on the particle should obey the force equilibrium condition as follows:

$$
F_{\mathrm{sw}}+F_{\mathrm{ap}}=F_{\mathrm{sp}}+F_{\mathrm{aw}}
$$

The water penetration depth $\delta_{\mathrm{w}}$ is mainly used to describe the removal rate in the CMP process. In most situations, plastic deformation occurs on the wafer surface, whereas elastic deformation occurs on the pad.

Elastic contact theory states that the force between the pad and the particle is given as follows [27]:

$$
F_{\mathrm{sp}}=\frac{4}{3} E_{\mathrm{sp}}\left(\frac{D}{2}\right)^{1 / 2} \delta_{\mathrm{p}}^{3 / 2}
$$

where $D$ is the particle diameter, and $E_{\mathrm{sp}}$ is the composite Young's modulus of the pad and the nanoparticle defined as follows:

$$
E_{\mathrm{sp}}=1 /\left(\frac{1-\mu_{\mathrm{p}}^{2}}{E_{\mathrm{p}}}+\frac{1-\mu_{\mathrm{s}}^{2}}{E_{\mathrm{s}}}\right)
$$

$E_{\mathrm{s}}$ and $\mu_{\mathrm{s}}$ are the Young's modulus and Poisson ratio, respectively, for the particle. $E_{\mathrm{p}}$ and $\mu_{\mathrm{p}}$ are both for the pad. 
(a)

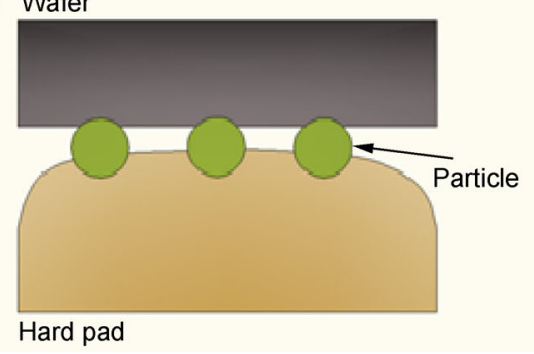

(b)

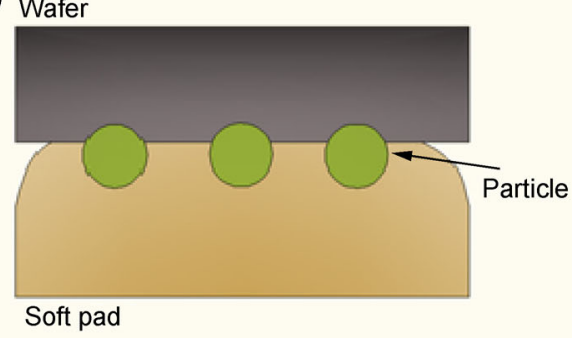

Fig. 2 Different geometries of contact states: (a) particle non-embedded and (b) particle embedded.

(a)

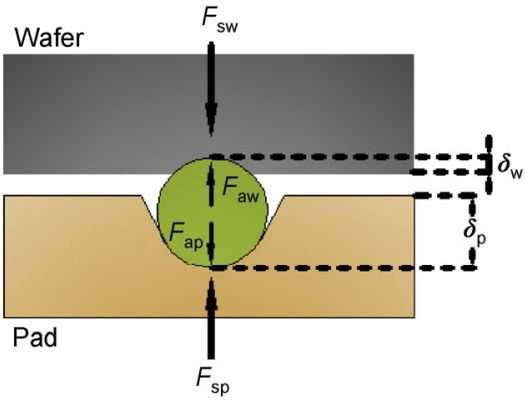

(b)

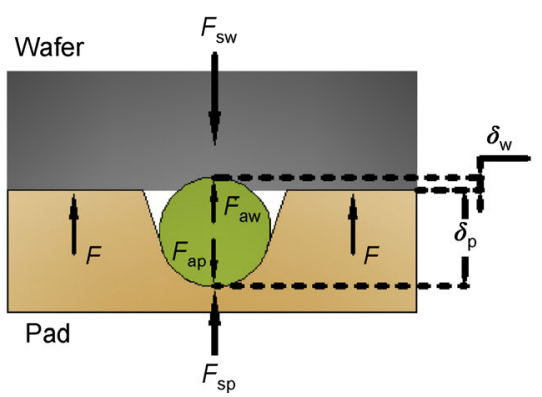

Fig. 3 Geometrical and mechanical behavior of the wafer, particle, and pad: (a) particle non-embedded and (b) particle embedded.

Plastic deformation theory states that the contact force between the wafer and the particle is given as follows [27]:

$$
F_{\mathrm{sw}}=H_{\mathrm{w}} \pi D \delta_{\mathrm{w}}
$$

where $H_{\mathrm{w}}$ is the wafer surface hardness.

A suction effect is observed from the pad for the adhesion force between the pad and the particles. Johnson, Kendall, and Roberts (JKR) theory could be used in this case because the Young's modulus of the pad is much smaller. The adhesion force is given through JKR theory as follows [28]:

$$
F_{\text {ap }} \approx 1.5 \pi W_{\text {ap }} r
$$

Here $W_{\text {ap }}$ is the thermodynamic work of adhesion between pad and particle, while $r$ is particle radius.

And DMT model is applied to obtain the adhesion force between particles and wafer. As both wafer and particles appear hard, DMT theory will be much more adapted, and the adhesion force is given as [28]

$$
F_{\mathrm{aw}} \approx 2 \pi W_{\mathrm{aw}} r
$$

where $W_{\mathrm{aw}}$ is the thermodynamic work of adhesion between the pad and the particle.

\subsection{Wafer and pad deformation}

In the CMP processes, the wafer penetration depth and pad deformation should obey the following geometrical condition if the contact state is particle embedded, as shown in Fig. 3(b):

$$
\delta_{\mathrm{w}}+\delta_{\mathrm{p}}=D
$$

The following equation is derived from Eqs. (1), (2), (4), (5), and (6):

$$
\frac{4}{3} E_{\mathrm{sp}}\left(\frac{D}{2}\right)^{1 / 2}\left(D-\delta_{\mathrm{w}}\right)^{3 / 2}+2 \pi W_{\mathrm{aw}} r=H_{\mathrm{w}} \pi D \delta_{\mathrm{w}}+1.5 \pi W_{\mathrm{ap}} r
$$

$\delta_{\mathrm{w}}$ can be obtained using the above-mentioned equations.

In the CMP processes, there is no geometrical condition for $\delta_{\mathrm{w}}$ and $\delta_{\mathrm{p}}$ if the contact state is particle non-embedded, as shown in Fig. 3(a). Hence, Eqs. (7) and (8) are unsuitable.

A simplified model is presented in the nonembedded condition to obtain the force on a single particle and estimate the number of particles in a unit nominal contact area. The research results by Xia and 
Ahmadi [26] stated that solid-solid contacts between the wafer and the pad asperities must be present to sustain the imposed load. However, not the entire wafer area will be in contact with the pad. Furthermore, the sum of the solid-solid contact area is the real contact area. The nominal polishing pressure $P$ is sustained by the real contact area. Hence, the real contact area $A_{\mathrm{r}}$ could be provided as follows:

$$
A_{\mathrm{r}}=\frac{P}{P_{\mathrm{r}}} A
$$

where $A$ is the nominal contact area equal to the wafer area and $P_{\mathrm{r}}$ is the real mean contact pressure. The real contact area ratio is then provided as follows:

$$
k_{\mathrm{r}}=\frac{A_{\mathrm{r}}}{A}=\frac{P}{P_{\mathrm{r}}}
$$

The real mean contact pressure between the pad and the wafer may be evaluated according to the pad topography and mechanics. The real contact area ratio varies from 0.00001 to 0.15 [11, 29]. The number of trapped particles depends on the ratio of the real contact area to the cross-sectional area of a particle. However, in practice, the particles do not fully cover the pad surface. Furthermore, the dimensionless parameter $C$ is used to specify the particle surface concentration with respect to the slurry. Therefore, the number of particles in a unit nominal contact area is provided as follows:

$$
N=\frac{C A_{\mathrm{r}}}{\pi r^{2}} \frac{1}{A}=\frac{A_{\mathrm{r}}}{A} \frac{C}{\pi r^{2}}=\frac{k_{\mathrm{r}} C}{\pi r^{2}}
$$

where $r$ is the particle radius and the value of $C$ is usually lower than 0.19 [18].

The force between the pad and the particle is then provided as follows:

$$
F_{\mathrm{sp}}=\frac{P}{N}=\frac{P \pi r^{2}}{k_{\mathrm{r}} \mathrm{C}}
$$

where $P$ is the polishing pressure between the pad and the wafer.

$\delta_{\mathrm{w}}$ is obtained using Eqs. (1), (4), (5), (6), and (12) as follows:

$$
\delta_{\mathrm{w}}=\left(\frac{P \pi r^{2}}{k_{\mathrm{r}} C}+2 \pi W_{\mathrm{aw}} r-1.5 \pi W_{\mathrm{ap}} r\right) / H_{\mathrm{w}} \pi D
$$

The contact radius is also evaluated as:

$$
a \approx \sqrt{2 r \delta_{\mathrm{w}}}
$$

\subsection{Contact state analysis}

Knowing the contact states of the wafer, pad, and particle in the CMP process is significant. The contact state should first be particle non-embedded when the polishing pressure is very low or the pad is relatively hard. This state may change to particle embedded as the polishing pressure increases. The pad and wafer deformation should obey Eq. (6) for a critical contact state from particle non-embedded to particle embedded. Substituting Eqs. (2) and (4) into Eq. (7) provides the following:

$$
\left(\frac{3}{4} \frac{F_{\mathrm{sp}}^{\mathrm{c}}}{E_{\mathrm{sp}}}\right)^{2 / 3}\left(\frac{2}{D}\right)^{1 / 3}+\frac{F_{\mathrm{sp}}^{\mathrm{c}}}{H_{\mathrm{w}} \pi D}=D
$$

$F_{\mathrm{sp}}^{\mathrm{c}}$ is the critical contact force on the particle. The adhesive force in Eq. (15) is neglected because $F_{\mathrm{sp}}^{\mathrm{c}}$ is much bigger than the force in most situations. The following formula is obtained by substituting Eq. (12) into Eq. (15):

$$
\left(\frac{3}{4} \frac{P_{\mathrm{th}} \pi}{E_{\mathrm{sp}} k_{\mathrm{r}} C}\right)^{2 / 3}+\frac{1}{4} \frac{P_{\mathrm{th}}}{H_{\mathrm{w}} k_{\mathrm{r}} C}=1
$$

where $P_{\text {th }}$ is the critical pressure between the pad and the wafer.

From Eq. (16), $P_{\mathrm{th}}$ is known to be independent of the particle radius. The contact state in a CMP process should be particle embedded when the polishing pressure is over the critical pressure; otherwise, the contact state should be particle non-embedded.

Two dimensionless parameters, $1.77\left(\frac{P}{E_{\mathrm{sp}} k_{\mathrm{r}} C}\right)^{2 / 3}$ and $0.25 \frac{P}{H_{\mathrm{w}} k_{\mathrm{r}} \mathrm{C}}$, can be taken for judging the contact state, as shown in Fig. 4.

Figure 4 shows the critical line with a slope of -1 . The area above the line demonstrates the particle embedded state, whereas the area below it shows the particle non-embedded state. 


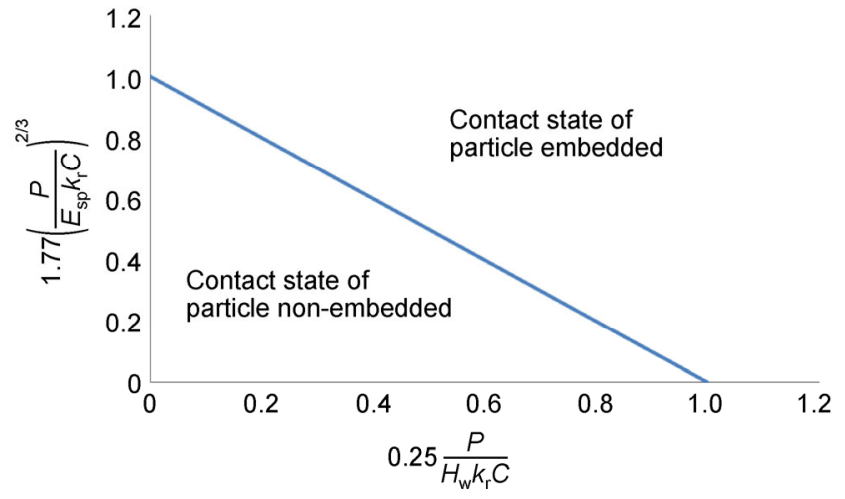

Fig. 4 Map of determination for the contact state.

\section{Different removal models}

The particle interaction with the wafer surface and the removal process for the wafer material are both complicated. Three models can be employed to evaluate the mechanical removal rate.

\subsection{Removal rate by abrasive wear}

The particles come into contact with the wafer surface. The penetration depth then occurs and slides nearly parallel to the wafer surface. The materials are then removed by abrasive wear.

The shadow area in Fig. 5 shows the removed material. The MRR by a single particle is provided as follows:

$$
\mathrm{MRR}=a \delta_{\mathrm{w}} U
$$

where $a$ is the contact radius; $\delta_{\mathrm{w}}$ is the penetration depth; and $U$ is the pad velocity.

\subsection{Removal rate by adhesive wear}

When two surfaces come into contact, their asperity

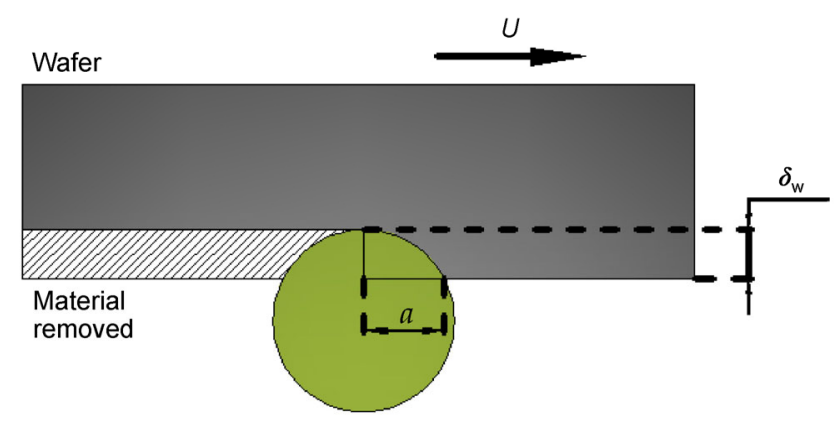

Fig. 5 Schematic of the removal model by abrasive wear. summits will probably adhere to each other because of the thermodynamic work and adhesion force. A break will probably occur within one of the materials when the contacts are broken during sliding or rolling. In a CMP process, such mechanical break sometimes occurs within the underneath wafer and not in the original particle and wafer interface. The wafer materials are then removed in this manner, as shown in Fig. 6.

Zhou and Pan [29] observed an atomic-scale smooth surface after a CMP process in their experiments. Therefore, it can be assumed that only one atomic layer is removed in a single adhesion process. The material removed by a single particle is then provided as follows:

$$
\operatorname{MRR}=k_{\mathrm{a}} \pi a^{2} d n
$$

where $d$ is the atomic diameter of the silicon, which is the wafer material; $a$ is the particle contact radius; and $k_{\mathrm{a}}$, which has a value generally below 0.11 , is the adhesive wear coefficient used to describe the probability of effective adhesion [30]. $n$ is the number of adhesions per second for a single particle, which is estimated as $n=\frac{U}{2 a}$. The following equation is then obtained:

$$
\mathrm{MRR}=k_{\mathrm{a}} \pi a^{2} d \frac{U}{2 a}=\frac{k_{\mathrm{a}} \pi}{2} a d U
$$

\subsection{Removal rate by erosive wear}

During a CMP process, part of the abrasive particles travels in the slurry and has the chance to impact the wafer surface $[31,32]$. Erosive wear then occurs. Figure 7 shows the impact process.

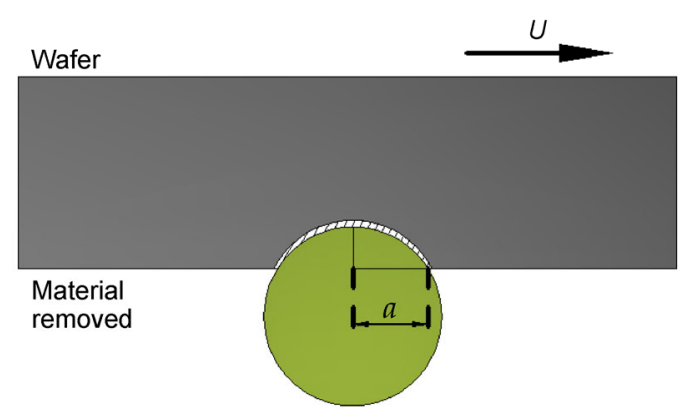

Fig. 6 Schematic of the removal model by adhesive wear. 


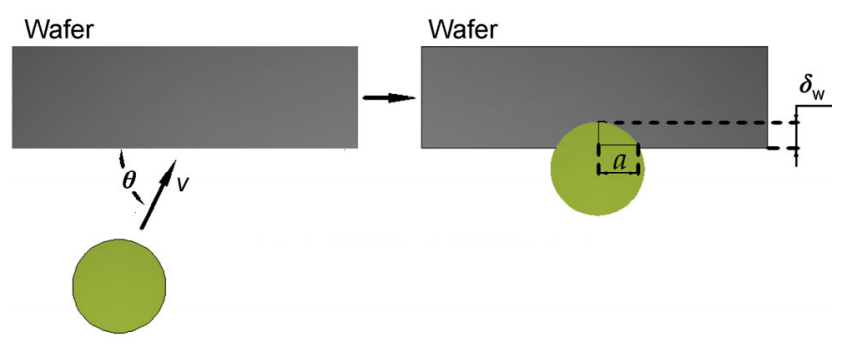

Fig. 7 Schematic of the removal model by erosive wear.

The impact of the abrasive particles during the erosive wear process also results in deformation and penetration into the surface. The ratio of the particle kinetic energy transferring to the strain energy is assumed to be $\eta$. The following equation is then obtained:

$$
E_{\text {strain }}=f\left(\delta_{\mathrm{w}}\right)=\frac{1}{2} m(v \sin \theta)^{2} \eta
$$

where $\theta$ is the impact direction angle, $m$ is the particle mass, and $v$ is the particle velocity. The energy conversion in the impact between the particles and the wafer could be approximately considered as rigidbody collisions. By estimating the energetic coefficient of restitution, it is assumed that less than $20 \%$ of the particle kinetic energy can transform to strain energy because of energy loss in the CMP process [33]. The penetration depth $\delta_{\mathrm{w}}$ is then acquired. The contact radius $a$ can be obtained using Eq. (14).

Similar to the adhesive wear process, the erosive wear removal rate for a single particle by each impact is estimated by

$$
\mathrm{MRR}=k_{\mathrm{e}} \pi a^{2} d n^{*}
$$

where $n^{*}$ is the number of impacts per second between the particle and the wafer, and $k_{\mathrm{e}}$ is the erosive wear coefficient. It is assumed that the manner by which the material here is removed is similar to that in the adhesive wear. Furthermore, the value of $k_{\mathrm{e}}$ will be a little smaller than $k_{\mathrm{a}}$ in Eq. (18). Some free particles in the slurry exhibit the reciprocating motion from the wafer to the pad [31]. A particle travels a distance of the length two times of the asperity summit height each time it impacts the wafer surface. Hence, the number of impacts per second between the particle and the wafer is estimated as follows:

$$
n^{*} \approx \frac{v}{2 R_{a}}
$$

where $v$ is the average particle traveling velocity, and $R_{\mathrm{a}}$ is the pad roughness. Notably, the kinetic energy of a particle will be partly dissipated after an impact. Nevertheless, the interaction force among the particles and surface and the fluid flow and pad motion may again speed up the particle [31]. Therefore, it is reasonable to assume that the particles can travel in the slurry at a stable average velocity after several times of impacts.

\section{Results and discussion}

Table 1 shows some fundamental parameters describing the CMP process conditions based on the actual working situations [27]. The wafer and abrasive particle materials are $\mathrm{Si}$ and $\mathrm{SiO}_{2}$, respectively. The thermodynamic work of adhesion is defined by the particle, wafer, and pad properties, and influenced by slurry conditions. The adhesion work between the wafer and the particle is assumed as $0.0141 \mathrm{~J}$ for silica [18]. The literature reveals the adhesion work between the pad and the particle to be $\sim 0.0081 \mathrm{~J}$ [34].

\subsection{Penetration depth of wafer by a single particle}

The Young's modulus of the hard polishing pad is set

Table 1 Parameters of the CMP process.

\begin{tabular}{lc}
\hline \multicolumn{1}{c}{ Parameters } & Value \\
\hline Polishing pressure, $P$ & $15-90 \mathrm{kPa}$ \\
Work of adhesion between wafer and particle, $W_{\text {aw }}$ & $0.0141 \mathrm{~J}$ \\
Work of adhesion between pad and particle, $W_{\mathrm{ap}}$ & $0.0081 \mathrm{~J}$ \\
Young's modulus of pad, $E_{\mathrm{p}}$ & 10 or $100 \mathrm{MPa}$ \\
Poisson ratio of pad, $\mu_{\mathrm{p}}$ & 0.22 \\
Young's modulus of wafer, $E_{\mathrm{w}}$ & $66 \mathrm{GPa}$ \\
Hardness of wafer, $H_{\mathrm{w}}$ & $2.8 \mathrm{GPa}$ \\
Poisson ratio of wafer, $\mu_{\mathrm{w}}$ & 0.3 \\
Young's modulus of particles, $E_{\mathrm{s}}$ & $72 \mathrm{GPa}$ \\
Poisson ratio of particles, $\mu_{\mathrm{s}}$ & 0.26 \\
Velocity of pad, $U$ & $0.5 \mathrm{~m} / \mathrm{s}$ \\
Concentration of slurry, $C$ & 0.1 \\
Real contact area ratio, $k_{\mathrm{r}}$ & 0.04 \\
\hline
\end{tabular}


as $100 \mathrm{MPa}$. Figure 4 shows that the contact state is particle non-embedded.

Figure 8 demonstrates the variations of the penetration depth with polishing pressure for different particle sizes when $E_{\mathrm{p}}=100 \mathrm{MPa}$ for a single particle. The penetration depth linearly increases with the polishing pressure. The big particle size for the slurries with the same particle concentration indicates less effective particles between the pad and wafer asperities. Therefore, the bigger the particle size at the same pressure, the larger the penetration depth into the wafer. Moreover, the load on a single particle will be bigger, which leads to deeper penetration. These results are consistent with those reported in the literature [18].

The Young's modulus for the soft pad is set as $10 \mathrm{MPa}$. The critical pressure obtained using Eq. (16) is $\sim 48 \mathrm{kPa}$. Therefore, the contact state for the polishing pressure below $48 \mathrm{kPa}$ is particle non-embedded. The contact state over that pressure is particle embedded. Figure 9 shows the penetration depth variations with polishing pressure.

Figure 9 also demonstrates that the penetration depth is not determined by the polishing pressure when the contact state is particle embedded. Accordingly, the penetration depth is only related with the particle diameter. Bigger particles lead to deeper penetration, which is also accompanied by larger contact forces on each particle.

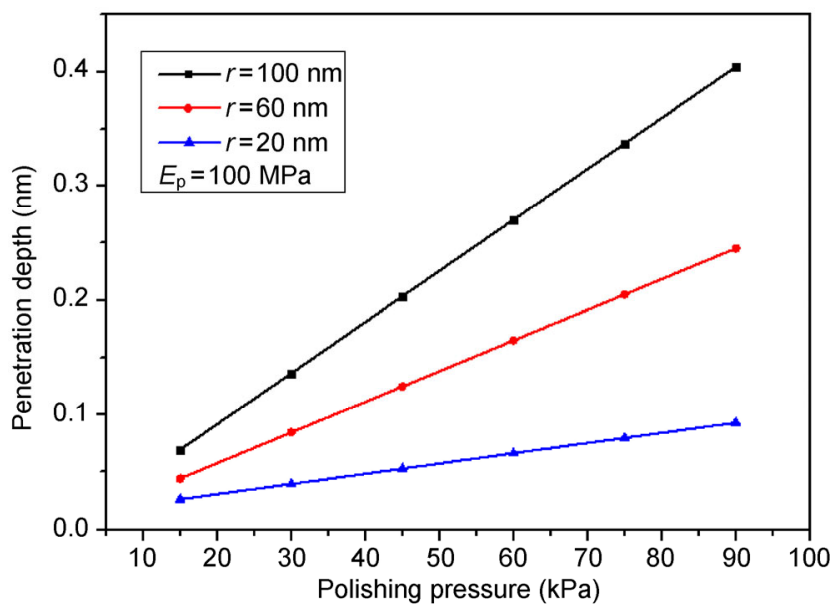

Fig. 8 Variations of the penetration depth with polishing pressure for different particle sizes $\left(E_{\mathrm{p}}=100 \mathrm{MPa}\right.$, particle non-embedded).

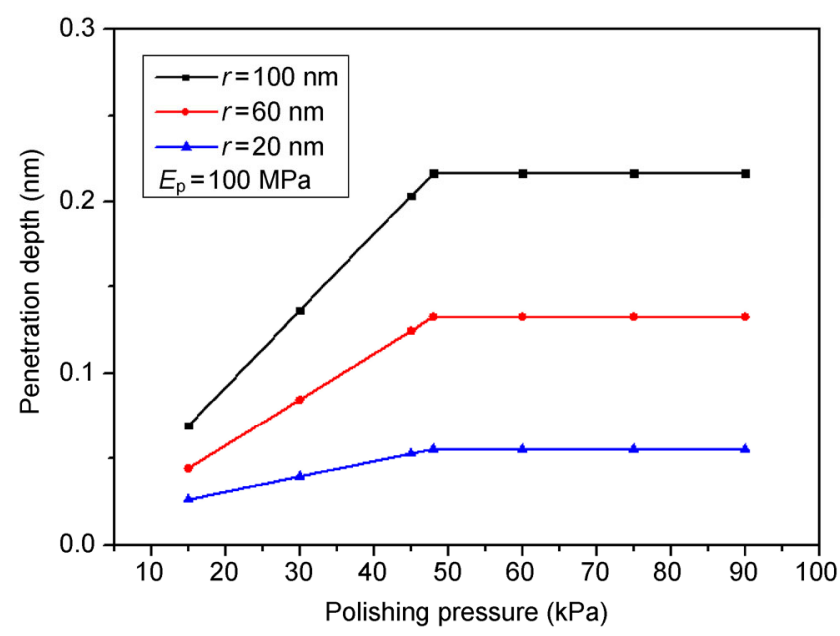

Fig. 9 Variations of the penetration depth with polishing pressure for different particle sizes $\left(E_{\mathrm{p}}=10 \mathrm{MPa}\right)$.

\subsection{Removal rate of by a single particle}

\subsubsection{Removal rate by abrasive wear}

The removal rate by a single particle is calculated and analyzed by using Eq. (17). The particle radii are set as $20 \mathrm{~nm}, 60 \mathrm{~nm}$, and $100 \mathrm{~nm}$. Figures 10 (hard pad) and 11 (soft pad) show the different removal rate variations with polishing pressure.

Figure 10 demonstrates that the removal rate for a hard pad increases with the polishing pressure because the contact state is always particle non-embedded, and the variations are non-linear. Meanwhile, the removal rate for the soft pad increases when the

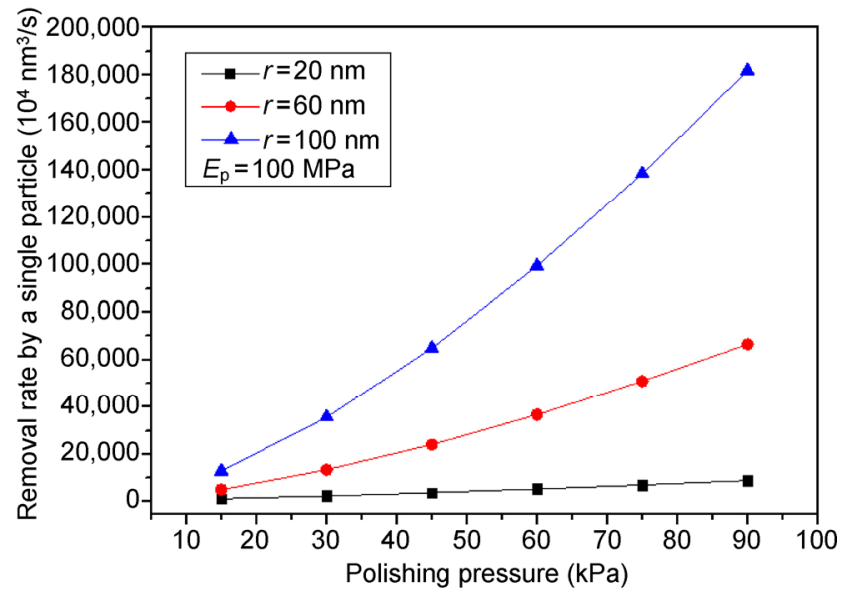

Fig. 10 Variations of the removal rate with polishing pressure by a single particle of abrasive wear (hard pad). 


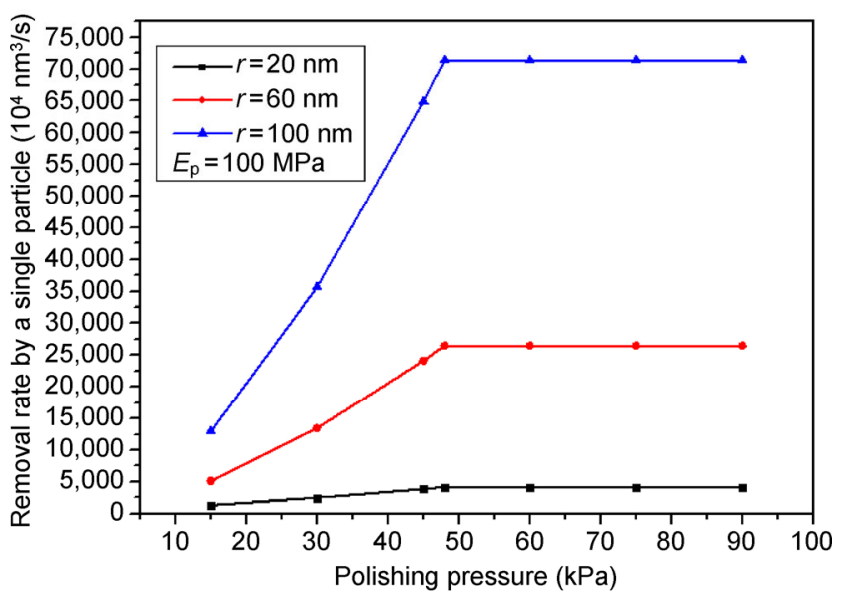

Fig. 11 Variations of the removal rate with polishing pressure by a single particle of abrasive wear (soft pad).

pressure is below $48 \mathrm{kPa}$. The removal rate then does not change with the polishing pressure increase because the contact state changes to particle embedded when the pressure is over $48 \mathrm{kPa}$. The penetration depth in this state is not determined by the polishing pressure.

\subsubsection{Removal rate by adhesive wear}

The removal rate value can be estimated using Eq. (19). Figures 12 and 13 show the results. The variation trend of the removal rate with the polishing pressure is similar to that of the abrasive wear. In addition, the removal rate is much smaller than that of abrasive wear under the same polishing pressure. The removal rate increases with the polishing pressure when the contact state is particle non-embedded. It also does not

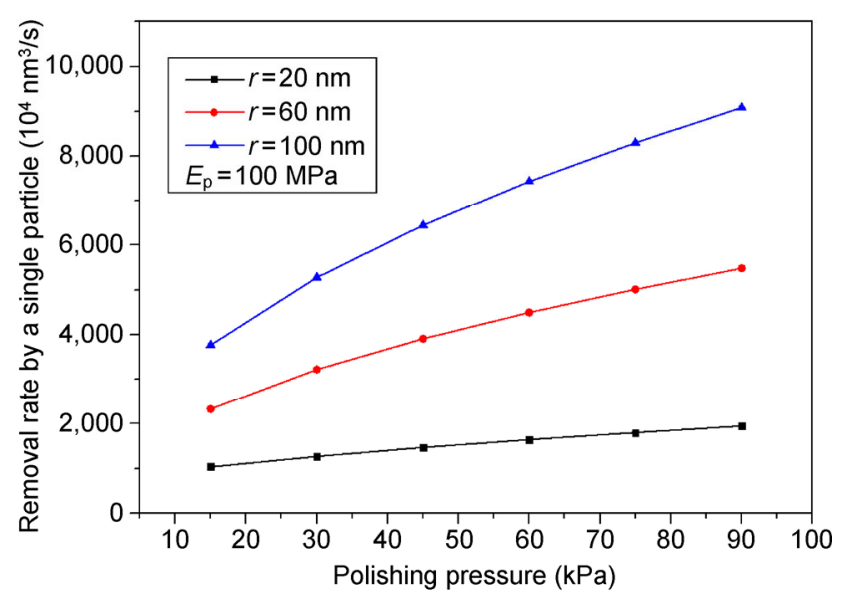

Fig. 12 Variations of the removal rate by a single particle of adhesive wear with polishing pressure (hard pad).

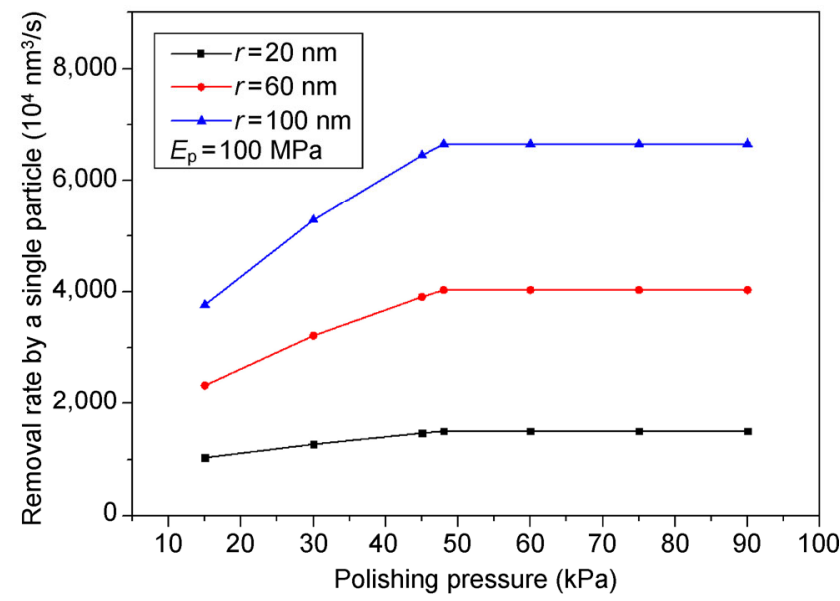

Fig. 13 Variations of the removal rate by a single particle of adhesive wear with polishing pressure (soft pad).

change when the contact state is particle embedded. A change of the contact state is observed in the soft pad. The larger-sized particles cause bigger removal rates.

\subsubsection{Removal rate by erosive wear}

The impact direction angle is set as $45^{\circ}$, and $\eta$ is assumed to be 0.2 [33]. The correspondences of wafer deformation and strain energy are obtained by calculation using the ANSYS workbench. These data are used to solve Eq. (20) and obtain the relationship between strain energy and penetration depth. The erosive depth variations with the particle traveling velocity are obtained as shown in Fig. 14 for the particles of $r=20 \mathrm{~nm}$ and $r=60 \mathrm{~nm}$.

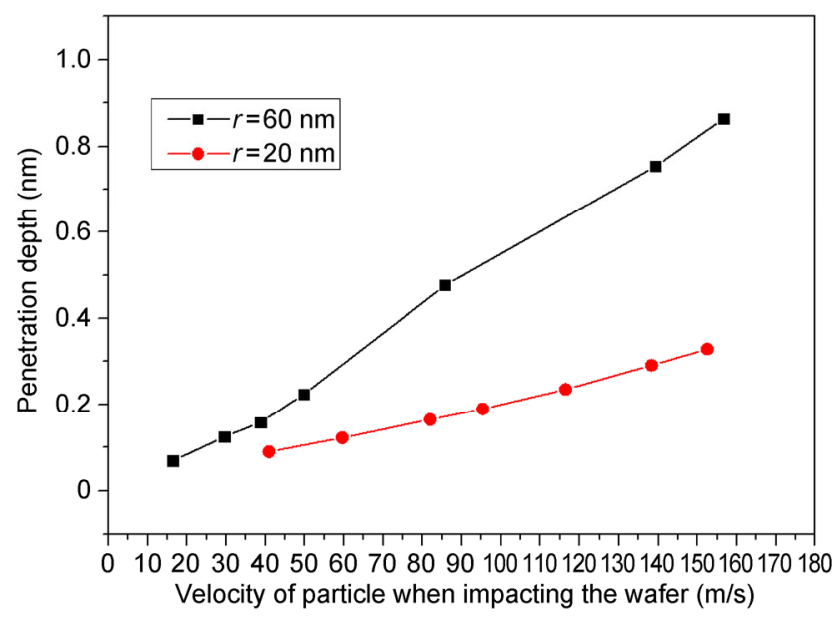

Fig. 14 Variations of the penetration depth with traveling particle velocity. 
The removal rate by impact can be estimated according to Eqs. (21) and (22) (Fig. 15). The pad roughness herein is assumed to be $20 \mu \mathrm{m}$.

Figure 15 shows that the bigger particle will cause deeper penetration and the larger removal rate for the same average velocity. The removal rate of erosive wear becomes a large value for the particle size of $60 \mathrm{~nm}$ when the particle velocity is over $60 \mathrm{~m} / \mathrm{s}$. However, the material removal process by erosive wear will not be continuous like those by cutting and adhesive wear. Therefore, the removal rate $f$ is smaller in most cases. However, the removal rate by cutting and adhesive wear will be extremely small when the polishing pressure is very small. The impact may then play a big role.

\subsection{Comparison of different removal models}

Figures 16 and 17 show the comparisons among abrasive, adhesive, and erosive wear for the particle radii of $20 \mathrm{~nm}$ and $60 \mathrm{~nm}$, respectively. The MRR ratio of adhesive wear to abrasive wear increases with the polishing pressure decrease because the penetration depth decreases for abrasive wear when the polishing pressure is small. The ratio will be larger under the same polishing pressure when the particle size is smaller. The removal rate of adhesion wear for a particle with a $20 \mathrm{~nm}$ radius can reach about $77 \%$ of that for abrasive wear. Therefore, the adhesive wear effect plays an increasingly more important role when the particle size or polishing pressure decreases.

The MRR of erosive wear is very small in most situations. However, this may be large and even come

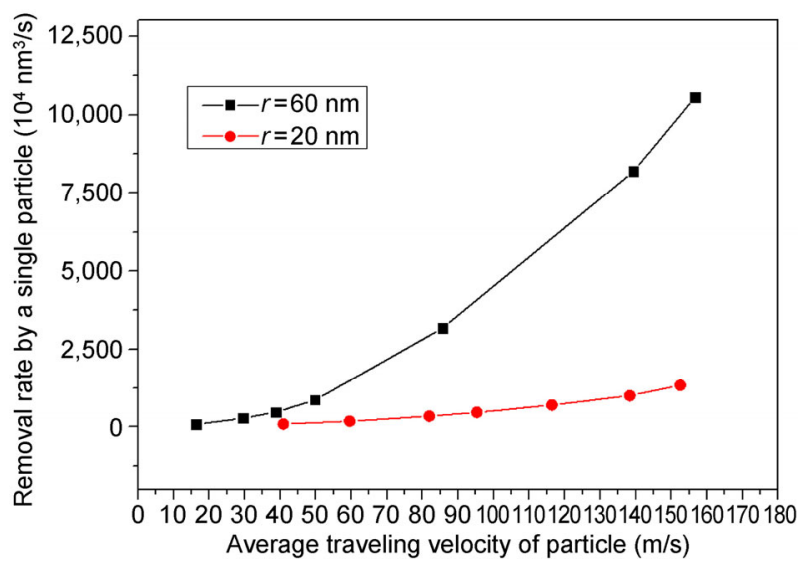

Fig. 15 Variations of the removal rate with average particle velocity.

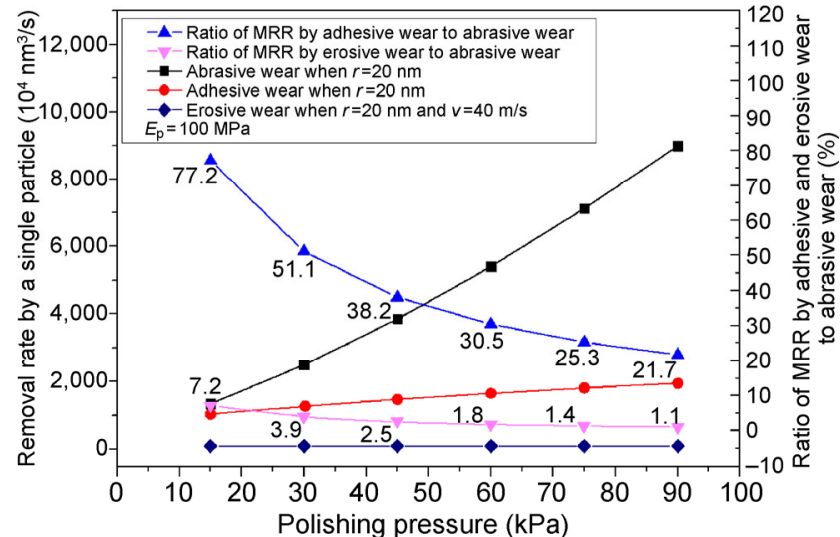

Fig. 16 Comparison among abrasive, adhesive, and erosive wear $(r=20 \mathrm{~nm})$.

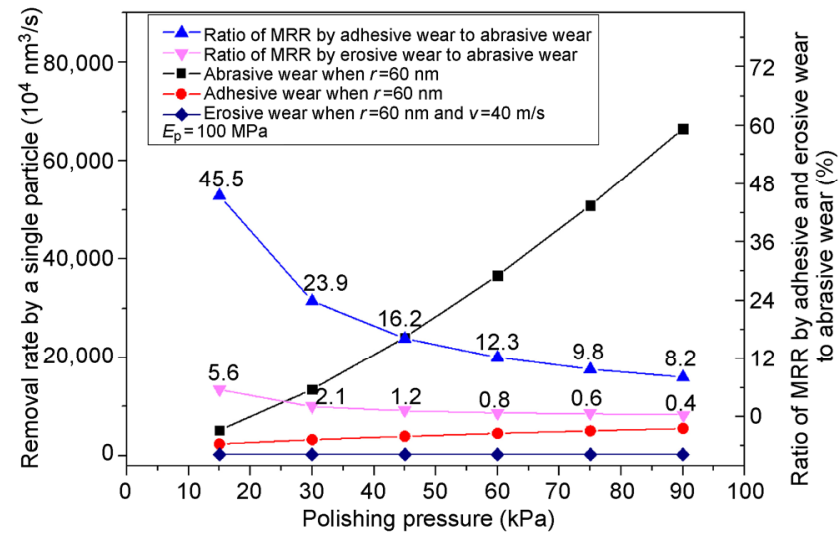

Fig. 17 Comparison among abrasive, adhesive, and erosive wear $(r=60 \mathrm{~nm})$.

close to the removal rates when the particle velocity is very high because of other wear forms. The average particle velocity in the calculations for Figs. 16 and 17 is set to $40 \mathrm{~m} / \mathrm{s}$. This value is much higher than the rotating velocity of the wafer in a real CMP process. However, Lei et al. [32] observed in a CMP experiment using a fluorescent technique that the particles move much faster than the rotating speed. Figures 16 and 17 also show that the ratio of MRR by erosive wear to abrasive wear comes close to $10 \%$ when the polishing pressure is below $20 \mathrm{kPa}$. The MRR caused by erosive wear is only related to the particle velocity, whereas that by abrasive and adhesive wear decreases with the polishing pressure decreases. The number of free particles, which could impact the wafer, may also be much more than the particles embedded in the pad. Therefore, it can be concluded that the effect of erosive wear may play an important role when the polishing pressure is very low. 


\section{Conclusions}

The interactions among the wafer, particle, and pad can be divided into the two types of contact states: particle embedded and particle non-embedded. Different contact states will affect the removal rate of CMP because of the different mechanical behaviors of the particles. This study first proposed a quantitative method of judging the contact state (Fig. 4, map of determination for the contact state). The critical pressure could help in determining the contact state and does not depend on the particle radius. The three following models of material removal are effective in the CMP process: abrasive, adhesive, and erosive wear. This paper compared the results for different models. Abrasive wear is usually the main part of material removal. The MRR by adhesive wear is about $10 \%$ to $50 \%$ of that by abrasive wear for a polishing pressure of over $30 \mathrm{kPa}$. The adhesive wear effect becomes more obvious when the pressure is lower. The removal rate by erosive wear may play an important role in the CMP process, especially for low polishing pressures. Only the MRR by a single particle is discussed herein. Studies on the whole MRR in a CMP process and the combined removal model of these three forms as well as the validation experiments will be performed and presented in future work.

\section{Acknowledgements}

We appreciate the financial support from the National Natural Science Foundation of China (Nos. 51375255, 91223202, 51321092).

Open Access: The articles published in this journal are distributed under the terms of the Creative Commons Attribution 4.0 International License (http://creativecommons.org/licenses/by/4.0/), which permits unrestricted use, distribution, and reproduction in any medium, provided you give appropriate credit to the original author(s) and the source, provide a link to the Creative Commons license, and indicate if changes were made.

\section{References}

[1] Tong Z. Present situation and future development of chemical mechanical polishing. Equipment for Electronic Products
33(6): 1-6 (2004)

[2] Gehman B L. In the age of $300 \mathrm{~mm}$ silicon, tech standards are even more crucial. Solid State Technol 44(8): 128-130 (2001)

[3] Dejule R. CMP challenges below a quarter micron. Semicond Int 20(13): 54-60 (1997)

[4] Preston F. The theory and design of plate glass polishing machines. Soc Glass Technol 11: 214-256 (1927)

[5] Warnock J. A two-dimensional process model for chemimechanical polish planarization. $J$ Electrocheml Soc 138(8): 2398-2402 (1991)

[6] Runnels S R, Eyman L M. Tribology analysis of chemicalmechanical polishing. J Electrochem Soc 141(6): 1698-1701 (1994)

[7] Tseng W T, Wang Y L. Re-examination of pressure and speed dependence of removal rate during chemical-mechanical polishing process. J Electrochem Soc 144(2): 15-17 (1997)

[8] Zhang F, Bunaina A. The role of particle adhesion and surface deformation in chemical mechanical polishing processes. Electrochemical and Solid-State Letters 1(4): 184-187 (1998)

[9] Shi F G, Zhao B. Modeling of chemical-mechanical polishing with soft pads. Appl Phys A 67(2): 249-252 (1998)

[10] Zhao B, Shi F G. Chemical mechanical polishing: Threshold pressure and mechanism. Electrochemical and Solid-State Letters 2(3): 145-147 (1999)

[11] Yu Y, Yu C C, Orlowski M. A statistical polishing pad model for chemical-polishing. In IEEE IEDM, Washington DC, 1993: 865-868.

[12] Liu C, Dai B, Tseng W. Modeling of the wear mechanism during chemical-mechanical polishing. J Electrochem Soc 143(2): 716-721 (1996)

[13] Zhang L, Tanaka H. Atomic scale deformation in silicon monocrystals induced by two-body and three-body contact sliding. Tribol Int 31(8): 424-433 (1998)

[14] Basse J L, Liang H. Probable role of abrasion in chemo chemical polishing of tungsten. Wear 233-235: 647-654 (1999)

[15] Zhao Y, Maietta D M, Chang L. An asperity microcontact model incorporating the transition from elastic deformation to fully plastic flow. J Tribol 122(1): 86-92 (2000)

[16] Luo J, Dornfled D A. Material removal mechanism in chemical mechanical polishing: Theory and modeling. IEEE Trans Semicond Manuf 14(2): 112-132 (2001)

[17] Zhao Y, Chang L. A micro-contact and wear model for chemical-mechanical polishing of silicon wafers. Wear 252(s3-4): 220-226 (2002)

[18] Ahmadi G, Xia X. A model for mechanical wear and abrasive particle adhesion during the chemical mechanical polishing process. J Electrochem Soc 148(3): G99-G109 (2001) 
[19] Fu G, Chandra A, Guha S, Subhash G. A plasticity-based model of material removal in chemical-mechanical polishing. IEEE Trans Semicond Manuf 14(4): 406-416 (2001)

[20] Wang Y, Zhao Y, An W, Wang J. Modeling the effects of cohesive energy for single particle on the material removal in chemical mechanical polishing at atomic scale. Appl Surf Sci 253(23): 9137-9141 (2007)

[21] Jeng Y R, Huang P Y. Impact of abrasive particles on the material removal rate in CMP. Electrochemical and SolidState Letters 7(2): 40-43 (2004)

[22] Jeng Y R, Huang P Y. A material removal rate model considering interfacial micro-contact wear behavior for chemical mechanical polishing. J Tribol 127(1): 190-196 (2005)

[23] Xu X, Luo J, Lu X, Zhang C, Guo D. Effect of nanoparticle impact on material removal. Tribol Trans 51(6): 718-722 (2008)

[24] Xu X, Luo J. Marangoni flow in an evaporating water droplet. Appl Phys Lett 91(12): 124102 (2007)

[25] Xu X, Luo J, Guo D. Nanoparticle-wall collision in a laminar cylindrical liquid jet. J Colloid Interf Sci 359(2): 334-338 (2011)

[26] Xia X, Ahamdi G. Surface removal rate in chemicalmechanical polishing. Particulate Science and Technology 20(3): 187-196 (2002)

[27] Si L, Guo D, Luo J, Lu X. Monoatomic layer removal mechanism in chemical mechanical polishing process: A

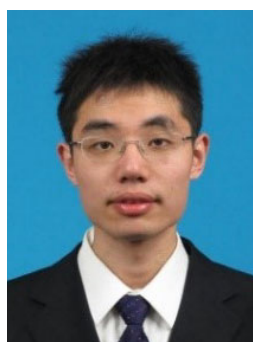

Hao CHEN. He received the bachelor degree in measurement techniques and instrumentation in 2013 from Tsinghua University, Beijing, China. After then, he is a postgraduate student in the State

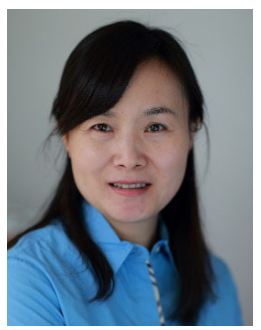

Dan GUO. She received the M.S. degree in engineering mechanics in 1995 from Xi'an Jiaotong University, Xi'an, China and Ph.D degree in engineering mechanics in 1999 from Tsinghua University, Beijing, China. She joined the State Key molecular dynamics study. J Appl Phys 107(6): 064310 (2010)

[28] Guo D, Li J N, Xie G X, Wang Y Y, Luo J B. Elastic properties of polystyrene nanosphere evaluated with atomic force microscopy: Size effect and error analysis. Langmuir 30(24): 7206-7212 (2014)

[29] Zhou Y, Pan G. Effects of ultra-smooth surface atomic step morphology on chemical mechanical polishing (CMP) performances of sapphire and $\mathrm{SiC}$ wafers. Tribol Int 87: 145-150 (2015)

[30] Rabinowicz E. Friction and Wear of Materials. John Wiley \& Sons, New York 1995.

[31] Lin G, Guo D, Xie G, Jia Q, Pan G. In situ observation of colloidal particle behavior between two planar surfaces. Colloids and Surfaces A-Physicochemical and Engineering Aspects 482: 656-661 (2015)

[32] Lei J, Guo D, Luo J, Pan G. Probing particle movement in cmp with fluorescence technique. J Electrocheml Soc 158(6): H681-H685 (2011)

[33] Yao W, Chen B, Liu C. Energetic coefficient of restitution for planar impact in multi-rigid-body systems with friction. Int J Impact Eng 31: 255-265 (2005)

[34] Seo J, Moon J, Bae J, Yoon K, Sigmund W, Paik U. Control of adhesion force between ceria particles and polishing pad in shallow trench isolation chemical mechanical planarization. J Nanosci Nanotechnol 14: 4351-4356 (2014)

Key Laboratory of Tribology at the same university. His research interests include measurement of nanomaterials mechanical properties and material removal mechanics in chemical mechanical polishing.

Laboratory of Tribology at Tsinghua University from 1999. Her current position is an associate professor and the deputy director of the laboratory. Her research areas cover the mechanism of interaction among nanoparticles and surface in ultra-smooth surface planarization, and the formation and failure of lubricant film in harsh conditions. 